\title{
BUFFER PROPERTIES OF SOIL MINERALS. PART 1. THEORETICAL ASPECTS
}

\author{
Igor Povar*, Oxana Spinu \\ Institute of Chemistry, Academy of Sciences of Moldova, 3, Academiei str., Chisinau MD 2028, Republic of Moldova \\ e-mail: ipovar@yahoo.ca; phone / fax: (+373 22) 739736
}

\begin{abstract}
The key quantitative characteristics of the theory of buffer action for polycomponent mono- and twophase systems have been derived. It is shown, that the buffer properties in relation to the solid phase components are amplified with an increase of solubility due to protolytic or complex formation equilibria in saturated solutions. It has been established, that the buffer capacities of components are mutually proportional, whereas for heterogeneous systems these relationships depend on the stoichiometric composition of solid phases. The deduced equations can be applied to the assessment of buffer action of the systems "natural mineral - soil solution", containing soluble and insoluble chemical species. A number of the important conclusions concerning the investigated buffer systems have been made. The obtained results are of interest for soil scientists and ecologists.
\end{abstract}

Keywords: buffer action, complex formation, soil solution, solid phase, thermodynamic stability.

\section{Introduction}

The capacity of buffer systems to oppose (resist) to the variation of their composition (usually to $\mathrm{pH}$ changes) by influence of external fluxes of chemical compounds of natural or anthropogenous character, that shift chemical equilibria, is called buffer property, and its efficiency - buffer action. The buffer action of soil is one of its fundamental physicochemical characteristics. The soil buffer action composes of the buffer action of a set of mineral and organic components, presented by solid, liquid and gaseous compounds. The buffer capacity of soils in relation to chemical compounds is defined by the content of chemical elements in the soil solution (the parameter of intensity) as well as by the content of mobile compounds of these elements in solid phases (the parameter of capacity). The buffer capacity of soils can be discovered by the fact that the increase of amounts of toxic metals (TM) is not accompanied by an increase of their content in plants; the different buffer action of the soils in relation to one element is manifested in unequal toxic concentrations for plants. The same soil can possess different buffer action in relation to different metals.

The chemical basis of the soil buffer capacity in relation to any chemical element is the nature of the equilibrium which is established between two groups of mobile compounds of an element. The analysis of the nature of buffer capacity of soils in relation to pollutants is essentially reduced to the analysis of laws of their absorption by soils. The more and more strongly the soil can keep pollutants, the more actively they go away from the soil solution into the composition of solid phase compounds, the better the soil resists to the increase of the concentration of polluting substances in its solution.

The problem of determination of the soil buffer capacity allows calculating the pollutant amounts which delivery does not essentially break the natural character of soil functioning, being the major one in the soil science, ecology and wildlife management. The complexity of the soil solution composition containing mineral phases and a large set of involved chemical compounds determine the possibility of simultaneous chemical reactions along with the capacity of solid phases of minerals to maintain relatively constant the aqueous solution composition. Under real conditions the buffer action of natural heterogeneous aqueous systems is expressed so as the consumption of any element from solution causes the partial dissolution of solid phases and as a result the composition of the solution is restored. Despite of an abundance of the information on buffer systems, the quantitative theory of buffer action has been developed only for mono-phase systems [1,2]. The buffer action of mono-phase buffers is usually based on protolytic equilibria between water, a weak acid or base or ampholytic compound and their conjugate pairs. The widespread use of buffers as well as the variety of chemical processes and phenomena associated with a certain acidity of solutions explains the constant interest in designing and studying new buffer systems.

Unlike the classical mono-phase buffer systems in which the buffer components are dissolved in a unique phase, in two-phase (heterogeneous) buffers they are distributed between two phases: in aqueous phase and solid (gaseous or liquid), insoluble phase. The aqueous (buffer) phase contains all the charged particles and a restricted quantity of electro-neutral species. The solid phase contains in significant quantities only electro-neutral particles and serves as their reservoir by means of which the equilibrium is adjusted and one of parameters of buffer system is maintained constantly [2-9]. The buffer action of two-phase systems is based on the shift of complex equilibrium, both homogeneous, and heterogeneous in the aqueous phase and between phases, respectively. By increasing the acidity of solution the role of simultaneous proceeding protolytic reactions with participation of salt anions increases; with an increase of alkalinity the contribution of complex formation reactions occurring with participation of salt cations amplifies. The humic acids and especially fulvic acids, occurring in soil solutions, essentially raise the 
solubility of metals and assist their moving in soils in the form of organic-mineral complexes, reducing thus the buffer action of soils in relation to heavy metal ions.

Authors [6] have proved that the buffer capacity is a special case of the sensitivity analysis, as a more general theoretical approach, which studies the answer of system to various external perturbations (as for example, the variation of the certain component concentration). We believe that it is more correct to consider, as a potential reservoir of buffer heterogeneous systems, the complex chemical heterogeneous equilibria with participation of solid phases [7]. Unfortunately, so far there are a small number of studies dedicated to the systematic investigations and the development of theoretical aspects of the buffer action for two-phase systems [1-9]. Besides, in the majority of these studies only the $\mathrm{pH}$ - buffer properties of heterogeneous systems were investigated. The concept of "buffer action" helps to find out which reactions control the composition of natural waters, including soil solutions [10-16]. The parameters of buffer action are integrated functions of all the soil chemical components by virtue of their capacity, by means of chemical reactions and sorption-desorption processes, to extinguish or strengthen the effect of entered pollutants [17].

Currently, extensive information on negative (harmful) transformations of soils, as a result of progressing acid and alkaline loads (in the form of mineral fertilizers, chemicals for protection of plants and industrial emissions dropping out with atmospheric precipitation) has been gathered. Thus, the quantitative assessment of the acid-base buffer action, revealing the degree of influence of the systematic use of fertilizers and technogenic pollution by substances of the acid and alkaline nature is an actual problem of the agrology. Besides, the buffer action of soils contains important information on the processes of soil formation (their orientation and intensity) which is used for the soil diagnostics and classification [17].

The aim of the present paper has been to develop the quantitative aspects of the buffer action of various components of homogeneous and heterogeneous systems and to establish their interrelation.

\section{Theory}

As a criterion for quantitative assessment of the intensity of buffer action of the studied multicomponent heterogeneous systems, one can use the value of the buffer capacity $\beta_{i}^{S}$ (the superscript index " $S$ " specifies the presence of solid phases), which can be defined as a partial derivative

$$
\beta_{i}^{S}=\left(\frac{\partial C_{i}^{0}}{\partial \ln [i]}\right)_{C_{j}^{0}(j \neq i)},
$$

where $C_{i}^{0}$ and [i] denote the initial (analytical) concentration in mixture and equilibrium concentration of the component " $i$ " of solid phase, correspondingly, at the same time the subscript index shows that the initial concentrations of other components of the mixture are maintained constant.

We will examine the process of formation of the sparingly soluble salt of arbitrary stoichiometric composition $M_{m} A_{n(S)}(M-$ metal ion, $A$ - anion of salt):

$$
M_{m} A_{n(S)}=m M+n A, \quad K_{S}=[M]^{m}[A]^{n} .
$$

The following set of possible simultaneous reactions in the saturated solution is taken into account:

$$
\begin{aligned}
& M+i H_{2} \mathrm{O}=M(\mathrm{OH})_{i}+i H, \quad K_{i}=\left[M(\mathrm{OH})_{i}\right][H]^{i} /[M] \\
& A+j H=H_{j} A, \quad K_{j}=\left[H_{j} A\right] /\left([A][H]^{j}\right) \\
& M+q A+r H=M H_{r} A_{q}, \quad K_{q}=\left[M H_{r} A_{q}\right] /\left([M][H]^{r}[A]^{q}\right) \\
& H_{2} \mathrm{O}=H+\mathrm{OH}, \quad K_{w}=[H][O H] .
\end{aligned}
$$

For the sake of simplicity, the charges of species are omitted. Near to the reaction equations the corresponding equilibrium constants are specified. The mass balance (MB) conditions in this system can be formulated by the following equations: 


$$
\begin{aligned}
& C_{M}^{0}=\Delta C_{M}+C_{M}^{r}=\Delta C_{M}+\sum_{i=1} \sum_{j=0} i\left[M_{i}(O H)_{j}\right]+\sum_{q=1} \sum_{r=0}\left[M H_{r} A_{q}\right] \\
& C_{A}^{0}=\Delta C_{A}+C_{A}^{r}=\Delta C_{A}+\sum_{l=0}\left[H_{l} A\right]+\sum_{q=1} \sum_{r=0} q\left[M H_{r} A_{q}\right] \\
& C_{H}^{0}=[H]-[O H]+\sum_{l=1} l\left[H_{l} A\right]-\sum_{i=1} \sum_{j=1} j\left[M_{i}(O H)_{j}\right]+\sum_{q=1} \sum_{r=1} r\left[M H_{r} A_{q}\right]
\end{aligned}
$$

The quantity $C_{i}^{r}$ represents the residual concentration in solution of the ion " $i$ ", e.g. the total concentration of all the species, containing a given ion, while $\Delta C_{i}$ is its molar quantity in the solid phase in $1 \mathrm{~L}$ of solution $[5-8,19,20]$. In the equations (8) $C_{H}^{0}$ denotes the excess of $H^{+}$ions in relation to hydroxyl ions in two-phase mixtures $\left(C_{H}^{0}=-C_{O H}^{0}\right)$. The square brackets designate the equilibrium concentrations of species in solution.

From the stoichiometric composition of the solid phase, the following ratio is obtained:

$\frac{\Delta C_{M}}{m}=\frac{\Delta C_{A}}{n}$ or $\Delta C_{A}=\frac{n}{m} \Delta C_{M}$

On the basis of the written equations it is possible to deduce the formulas for calculating the buffer capacity in relation to any component of the mixture. Firstly, the analysis of the deduction of the expression for the buffer capacity towards the metal ion (or the metal ion buffer capacity) will be examined. Taking the logarithm and differentiating the expression for the solubility product (1) on $\ln [M]$, one can find:

$$
\left(\frac{\partial \ln K_{S}}{\partial \ln [M]}\right)_{C_{H}^{0}, C_{A}^{0}}=0=m+n \frac{\partial \ln [A]}{\partial \ln [M]} \text {, and } \frac{\partial \ln [A]}{\partial \ln [M]}=-\frac{m}{n} \text {. }
$$

The subscript indices symbolize the conditions $C_{H}^{0}=$ const, $C_{A}^{0}=$ const, which are further omitted. Differentiating the MB equation (6) on $\ln [M]$, one can obtain the partial derivative:

$$
\begin{aligned}
& \left(\frac{\partial C_{M}^{0}}{\partial \ln [M]}\right)_{C_{H}^{0}, C_{A}^{0}} \equiv \beta_{M}^{S}=\frac{\partial \Delta C_{M}}{\partial \ln [M]}+\sum_{i=1} \sum_{j=0} i^{2}\left[M_{i}(O H)_{j}\right]+\sum_{q=1} \sum_{r=0}\left[M H_{r} A_{q}\right]+ \\
& +\frac{\partial \ln [H]}{\partial \ln [M]}\left(-\sum_{i=1} \sum_{j=1} i j\left[M_{i}(O H)_{j}\right]+\sum_{q=1} \sum_{r=0} r\left[M H_{r} A_{q}\right]\right)-\frac{m}{n} \sum_{q=1} \sum_{r=0} q\left[M H_{r} A_{q}\right]
\end{aligned}
$$

The deduced equation (11) contains two unknown terms:

$$
\beta_{M}^{S}=f\left(\frac{\partial \ln [H]}{\partial \ln [M]}, \frac{\partial \Delta C_{M}}{\partial \ln [M]}\right)
$$

For their determination, the MB equations (7) and (8) are differentiated on $\ln [M]$ :

$$
\begin{aligned}
& \left(\frac{\partial C_{A}^{0}}{\partial \ln [M]}\right)_{C_{M}^{0}, C_{H}^{0}}=0=\frac{n}{m} \frac{\partial \Delta C_{M}}{\partial \ln [M]}+\frac{\partial \ln [H]}{\partial \ln [M]}\left(\sum_{l=1} l\left[H_{l} A\right]+\sum_{q=1} \sum_{r=0} r q\left[M H_{r} A_{q}\right]\right)- \\
& -\frac{m}{n} \sum_{l=0}\left[H_{l} A\right]-\frac{m}{n} \sum_{q=1} \sum_{r=0} q^{2}\left[M H_{r} A_{q}\right]+\sum_{q=1} \sum_{r=0} q\left[M H_{r} A_{q}\right],
\end{aligned}
$$


whence

$$
\begin{aligned}
& \frac{\partial \Delta C_{M}}{\partial \ln [M]}=-\frac{m}{n} \frac{\partial \ln [H]}{\partial \ln [M]}\left(\sum_{l=1} l\left[H_{l} A\right]+\sum_{q=1} \sum_{r=0} r q\left[M H_{r} A_{q}\right]\right)+ \\
& \frac{m^{2}}{n^{2}} \sum_{l=0}\left[H_{l} A\right]+\frac{m^{2}}{n^{2}} \sum_{q=1} \sum_{r=0} q^{2}\left[M H_{r} A_{q}\right]-\frac{m}{n} \sum_{q=1} \sum_{r=0} q\left[M H_{r} A_{q}\right] .
\end{aligned}
$$

After that,

$$
\begin{aligned}
& \left(\frac{\partial C_{H}^{0}}{\partial \ln [M]}\right)_{C_{M}^{0}, C_{A}^{0}}=0=\frac{\partial \ln [H]}{\partial \ln [M]}\left([H]+[O H]+\sum_{l=1} l^{2}\left[H_{l} A\right]+\sum_{i=1} \sum_{j=0} j^{2}\left[M_{i}(O H)_{j}+\sum_{q=1} \sum_{r=1} r^{2}\left[M H_{r} A_{q}\right]\right)-\right. \\
& -\sum_{i=1} \sum_{j=0} i j\left[M_{i}(O H)_{j}\right]-\frac{m}{n} \sum_{l=0} l\left[H_{l} A\right]+\sum_{q=1} \sum_{r=1} r\left[M H_{r} A_{q}\right]-\frac{m}{n} \sum_{q=1} \sum_{r=1} r q\left[M H_{r} A_{q}\right]
\end{aligned}
$$

From the equation (13), it follows:

$$
\frac{\partial \ln [H]}{\partial \ln [M]}=\frac{\sum_{i=1} \sum_{j=0} i j\left[M_{i}(O H)_{j}\right]+\frac{m}{n} \sum_{l=1} l\left[H_{l} A\right]-\sum_{q=1} \sum_{r=1} r\left[M H_{r} A_{q}\right]+\frac{m}{n} \sum_{q=1} \sum_{r=1} r q\left[M H_{r} A_{q}\right]}{[H]+[O H]+\sum_{l=1} l^{2}\left[H_{l} A\right]+\sum_{i=1} \sum_{j=0} j^{2}\left[M_{i}(O H)_{j}\right]+\sum_{q=1} \sum_{r=1} r^{2}\left[M H_{r} A_{q}\right]}
$$

Substituting the expression (14) in the equation (13), after some rearrangements, one can obtain:

$$
\begin{aligned}
& \frac{\partial \Delta C_{M}}{\partial \ln [M]}=-\frac{m}{n}\left(\frac{\sum_{i=1} \sum_{j=0} i j\left[M_{i}(O H)_{j}\right]+\frac{m}{n} \sum_{l=1} l\left[H_{l} A\right]-\sum_{q=1} \sum_{r=1} r\left[M H_{r} A_{q}\right]+\frac{m}{n} \sum_{q=1} \sum_{r=1} r q\left[M H_{r} A_{q}\right]}{[H]+[O H]+\sum_{l=1} l^{2}\left[H_{l} A\right]+\sum_{i=1} \sum_{j=0} j^{2}\left[M_{i}(O H)_{j}\right]+\sum_{q=1} \sum_{r=1} r^{2}\left[M H_{r} A_{q}\right]}\right) \times \\
& \times\left(\sum_{l=1} l\left[H_{l} A\right]+\sum_{q=1} \sum_{r=0} r q\left[M H_{r} A_{q}\right]\right)+\frac{m^{2}}{n^{2}} \sum_{l=0}\left[H_{l} A\right]+\sum_{q=1}\left(\frac{m^{2} q^{2}}{n^{2}}-\frac{m q}{n}\right) \sum_{q=1} \sum_{r=0}\left[M H_{r} A_{q}\right]
\end{aligned}
$$

Further, substituting the obtained expressions for $\partial \ln [H] / \partial \ln [M](14)$ and $\partial \Delta C_{M} / \partial \ln [M]$ (15) into the equation (11), after a series of transformation, one can finally get:

$$
\begin{aligned}
& \beta_{M}^{S}=-\frac{\left(\sum_{i=1} \sum_{j=0} i j\left[M_{i}(O H)_{j}\right]+\frac{m}{n} \sum_{l=1} l\left[H_{l} A\right]-\sum_{q=1} \sum_{r=1} r\left[M H_{r} A_{q}\right]+\frac{m}{n} \sum_{q=1} \sum_{r=1} r q\left[M H_{r} A_{q}\right]\right)^{2}}{[H]+[O H]+\sum_{l=1} l^{2}\left[H_{l} A\right]+\sum_{i=1} \sum_{j=0} j^{2}\left[M_{i}(O H)_{j}\right]+\sum_{q=1} \sum_{r=1} r^{2}\left[M H_{r} A_{q}\right]}+ \\
& +\frac{m^{2}}{n^{2}} \sum_{l=0}\left[H_{l} A\right]+\sum_{q=1}\left(\frac{m^{2} q^{2}}{n^{2}}-2 \frac{m q}{n}+1\right) \sum_{q=1} \sum_{r=0}\left[M H_{r} A_{q}\right]+\sum_{i=1} \sum_{j=0} i^{2}\left[M_{i}(O H)_{j}\right]
\end{aligned}
$$

or

$$
\beta_{M}^{S}=\varphi_{3}-\frac{\varphi_{1}^{2}}{\varphi_{2}},
$$

where $\varphi_{1}, \varphi_{2}$ and $\varphi_{3}$ denote the following concentration functions:

$$
\varphi_{1}=\sum_{i=1} \sum_{j=0} i j\left[M_{i}(O H)_{j}\right]+\frac{m}{n} \sum_{l=1} l\left[H_{l} A\right]-\sum_{q=1} \sum_{r=1} r\left[M H_{r} A_{q}\right]+\frac{m}{n} \sum_{q=1} \sum_{r=1} r q\left[M H_{r} A_{q}\right]
$$




$$
\begin{aligned}
& \varphi_{2}=[H]+[O H]+\sum_{l=1} l^{2}\left[H_{l} A\right]+\sum_{i=1} \sum_{j=0} j^{2}\left[M_{i}(O H)_{j}\right]+\sum_{q=1} \sum_{r=1} r^{2}\left[M H_{r} A_{q}\right] \\
& \varphi_{3}=\frac{m^{2}}{n^{2}} \sum_{l=0}\left[H_{l} A\right]+\sum_{q=1}\left(\frac{m^{2} q^{2}}{n^{2}}-2 \frac{m q}{n}+1\right) \sum_{q=1} \sum_{r=0}\left[M H_{r} A_{q}\right]+\sum_{i=1} \sum_{j=0} i^{2}\left[M_{i}(O H)_{j}\right]
\end{aligned}
$$

Similarly, it is possible to prove that, for the buffer capacity towards proton, the following expression is valid:

$$
\left(\frac{\partial C_{H}^{0}}{\partial \ln [H]}\right)_{C_{M}^{0}, C_{A}^{0}} \equiv \beta_{H}^{S}=\varphi_{2}-\frac{\varphi_{1}^{2}}{\varphi_{3}} .
$$

For the buffer capacity towards the anion of the solid phase one can deduce:

$$
\beta_{A}^{S}=\frac{n^{2}}{m^{2}} \varphi_{3}-\frac{n^{2}}{m^{2}} \frac{\varphi_{1}^{2}}{\varphi_{2}}=\frac{n^{2}}{m^{2}}\left(\varphi_{3}-\frac{\varphi_{1}^{2}}{\varphi_{2}}\right)=\frac{n^{2}}{m^{2}} \beta_{M}^{S}
$$

On the basis of obtained equations (17), (19) and (20) the following remarkable conclusion follows: the buffer capacities towards different components are reciprocally proportional, while the buffer capacities in relation to the ions of the solid phase are interconnected through its stoichiometric coefficients:

$$
\frac{\beta_{A}^{S}}{n^{2}}=\frac{\beta_{M}^{S}}{m^{2}}
$$

It is worthy to mention that the obtained relations are only valid in the presence of the mineral (solid phase) $M_{m} A_{n(S)}$. The thermodynamic stability area of the latter is determined by the value of the Gibbs energy of the overall process (1)-(5) [5, 21-23]:

$$
\Delta G_{S, t o t}=-m R T \ln \frac{C_{M}^{r}}{C_{M}^{0}}-n R T \ln \frac{C_{A}^{r}}{C_{A}^{0}}
$$

The solid-phase is stable if $\Delta G_{S, t o t}>0$. The condition $\Delta G_{S, t o t}=0$ corresponds to the beginning of its dissolution and (or) sedimentation.

The analysis of the derived equations shows that the buffer capacities $\beta_{i}^{S}$ grow with the increase of the precipitate solubility, e.g. by rising the residual concentration of the component of minerals.

\section{Conclusions}

On the basis of the method of residual concentrations, the original mathematical expressions for the quantitative estimation of buffer capacities towards both components of the mineral phase were deduced.

The quantitative bases of the theory of buffer action for heterogeneous systems are stated. It is established, that buffer properties in relation to components of the solid phase amplify with the increase of the solubility. The buffer capacity of any component is a complex function of the equilibrium composition of multicomponent heterogeneous systems.

It is proved that the buffer capacities of components are mutually proportional and for heterogeneous systems these relations depend on the stoichiometric composition of the solid phases (minerals).

The use of results of this research allows predicting the variations of the composition and response of the soil environment at the technogenic loads increasing.

\section{Acknowledgments}

This work was supported by the Joint Operational Programme "Black Sea Basin 2007-2013”.

\section{References}

[1] Perrin, D.D.; Dempsey, B. Buffers for pH and metal ion control; Chapman and Hall: London, 1974; $175 \mathrm{p}$.

[2] Komari, N.P. Chemical metrology. Ionic heterogeneous equilibria; Vishcha Shkola: Kharkov, 1984; 208p. (Rus.) 
[3] Charykov, A.K.; Osipov, N.N. Carbonic acids and carboxylate complexes in chemical analysis; Khimia: Leningrad, 1991; pp. 204-207. (Rus.)

[4] Pfendt, L.B. Analyst. 1995, 120, pp. 2129 - 2144.

[5] Povar, I.; Rusu, V. Can. J. Chem. 2012, 90, pp. 395 - 402.

[6] Fishtik, I.; Povar, I. Can. J. Chem. 2006, 84, pp. 1036 - 1044.

[7] Povar, I. Russ. J. Inorg. Chem. 2000, 45, pp. 1632 - 1636, (Engl. Transl.).

[8] Povar, I. Russ. J. Inorg. Chem. 1997, 42, pp. 607-612, (Engl. Transl.).

[9] Povar, I.; Luca, C. Rev. Chim. 2003, 54, pp. 312-316, (Rom.).

[10] Van Breemen, N.; Wielemaker, W.G. Soil Sci. Soc. Amer. J. 1974, 38, pp. 55-60.

[11] Stumm, W.; Morgan, J.J. Aquatic Chemistry; Wiley; New York, 1981; 113 p.

[12] Filep, D.; Radly, M. Pochvovedenie. 1989, 12, pp. 48 - 59, (Rus.).

[13] Lozovik, P.A.; Potapova, I. Yu.; Bantsevich, T.V. Geochem. Int. 2007, 45, pp. 938 - 944, (Engl. Transl.).

[14] Langmuir, D. Aqueous Environmental Geochemistry; New Jersey: Prentice Hall, 1997; 600 p.

[15] Poznyak, S.P.; Gamkalo, M.Z. Pochvovedenie. 2001, 6, pp. 660 - 669, (Rus.).

[16] Sokolova, T.A.; Motuzova, G.V.; Malinina, M.S.; Obukhovskaya T.D. Chemical basis of soil buffer action; MGU: M., 1991; 106 p., (Rus.).

[17] Zaytseva, T.F. Izv. SO AN SSSR. Ser. biol. Nauk. 1987, 14, pp. 69-80, (Rus.).

[18] Povar, I. J. Anal. Chem. 1998, 53, pp. 1113 - 1119.

[19] Beresnev, E.N. Method of residual concentrations; Nauka: Moscow, 1992; 110 p., (Rus.).

[20] Fishtik, I.; Povar, I. Russ. J. Gen. Chem. 1987, 57, pp. 25 - 30, (Engl. Transl.).

[21] Povar, I. Ukr. Khim. Zh. 1994, 60, pp. 371 - 378, (Rus.).

[22] Povar, I.; Rusu, V. Can. J. Chem. 2012, 90, pp. 326 - 332. 